\title{
Digital Ulcers as an Initial Manifestation of Systemic Lupus Erythematosus
}

\author{
Edoardo Rosato, Ilenia Molinaro, Simonetta Pisarri and Felice Salsano
}

\begin{abstract}
Digital ulcers (DUs) and gangrene are common skin manifestations of connective tissue diseases, especially systemic sclerosis. Raynaud's phenomenon (RP) is an almost universal manifestation of systemic sclerosis, with 95\% of all patients being affected, and resulting in DUs in approximately 30\% of the patients each year. Although RP may be present in approximately $25-50 \%$ of the patients, DUs are relatively rare in systemic lupus erythematosus (SLE) and never present as an initial manifestation of disease. In this case report DUs appear as the initial manifestation of SLE in a young woman with a capillaroscopic scleroderma pattern and elevated systolic pulmonary arterial pressure.
\end{abstract}

Key words: systemic lupus erythematosus, digital ulcers, Raynaud's phenomenon, antiphospholipid syndrome

(Intern Med 50: 767-769, 2011)

(DOI: 10.2169/internalmedicine.50.4617)

\section{Introduction}

The term Raynaud's phenomenon (RP) is used to describe these episodic events, which represent vasoconstriction of the digital arteries, precapillary arterioles and cutaneous arteriovenous shunts. It typically starts in one or several digits after exposure to the cold or a stressful situation and then spreads symmetrically to all fingers of both hands. The prevalence of RP in the general population is approximately $3-5 \%$. It is classified as primary or secondary, according to the absence or the presence of a known associated disease, respectively. Secondary RP is frequently present in patients affected by connective tissue diseases (CTD), including systemic sclerosis (SSc), systemic lupus erythematosus (SLE), Sjögren's syndrome, dermatomyositis or polymyositis, and rheumatoid arthritis (1).

Digital ulcers (DUs) and gangrene are common skin manifestations of connective tissue diseases, especially systemic sclerosis. RP is an almost universal manifestation of SSc, with $95 \%$ of all patients being affected, and resulting in DUs in approximately $30 \%$ of the patients each year (2).

Although the RP may be present in approximately 25$50 \%$ of the patients, DUs are relatively rare in SLE and they do not represent the initial manifestation of SLE (3-5). We describe here a patient with DUs and Raynaud phenomenon as the initial presentation of SLE.

\section{Case Report}

A 21-year-old Caucasian woman was admitted to our Unit of Clinical Immunology in May 2008 when she presented with RP which began 3 weeks previously, DUs and edema of both hands associated with high-titre anti-nuclear antibodies (ANA: 1 : 640 homogeneous pattern). Doublestranded DNA (dsDNA) antibodies, antiextractable nuclear antigen autoantibodies (anti-ENA), anticentromere (ACA) antibodies, antiphospholipid (APL) antibodies (IgG and $\mathrm{IgM}$ ), complement $\mathrm{C} 3$ and $\mathrm{C} 4$, rheumatoid factor, anti-cyclic citrullinated peptide (anti-CCP) antibodies, antineutrophil cytoplasmic antibodies, the erythrocyte sedimentation rate (ESR), C-reactive protein (CRP), urinalysis, plasma levels of blood urea nitrogen, creatinine were all normal. The nailfold videocapillaroscopy (NVC) of the second, third, fourth and fifth finger showed frequent giant capillaries, frequent capillary haemorrhages, moderate loss of capillaries, mild disorganization of the capillary architecture, mild ramified capillaries. According to Cutolo et al (6) the capillaroscopic pat- 

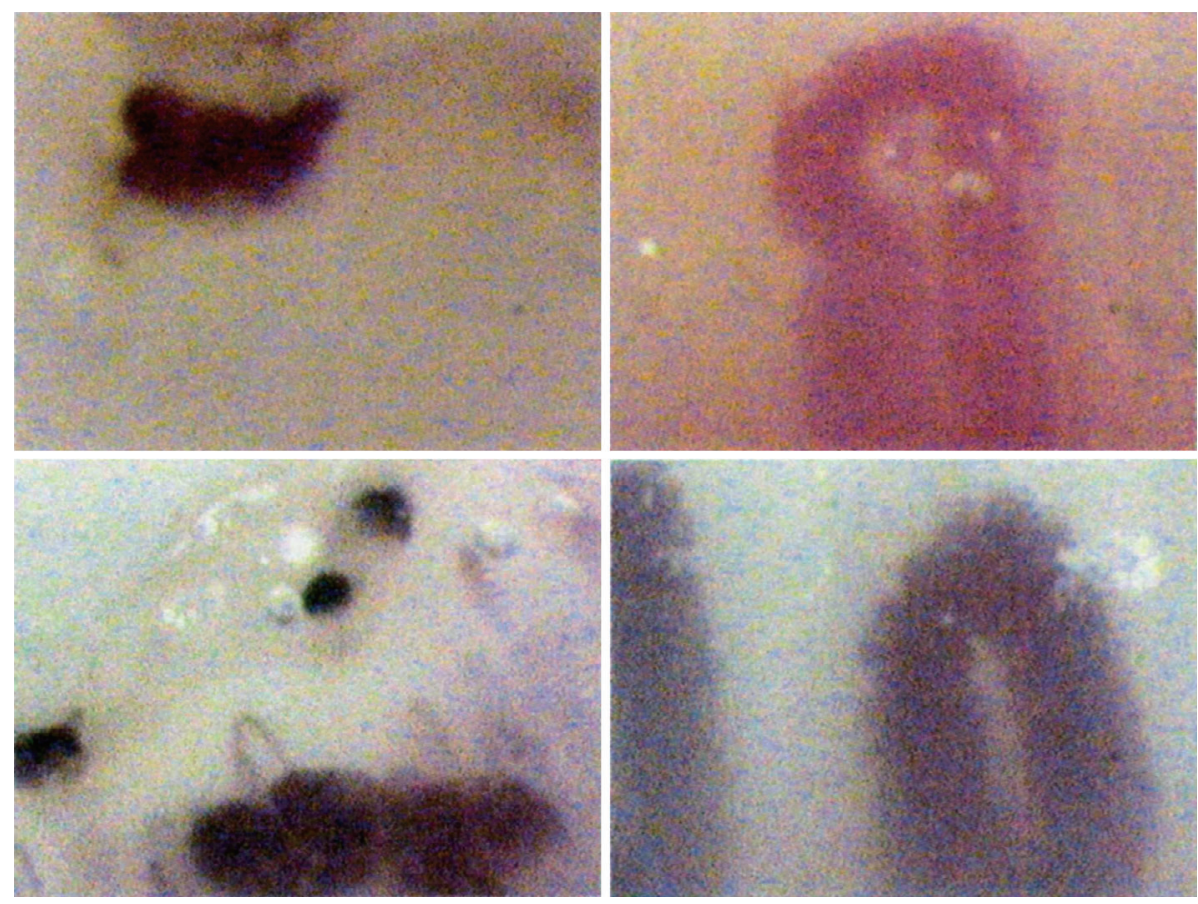

Figure 1. Nailfold videocapillaroscopy shows frequent giant capillaries, frequent capillary hemorrhages, moderate loss of capillaries, and mild disorganization of the capillary architecture.

tern identified within the "active SSc pattern" (Fig. 1). She had no skin involvement (tightness, thickening, non-pitting induration). The spirometry and diffusing capacity of carbon monoxide were normal, but the rest transthoracic echocardiogram (TTE) showed a mildly elevated pulmonary artery systolic pressure $(32 \mathrm{mmHg})$. Radiography and high resolution computed tomography (HRCT) of chest excluded the presence of interstitial pneumonia.

In order to improve the peripheral perfusion, she was treated with calcium channel blockers (nifedipine $30 \mathrm{mg}$ / day) and intravenous infusion of $\mathrm{N}$-acetylcysteine at a dosage of $15 \mathrm{mg} / \mathrm{kg} / \mathrm{h}$ for 5 consecutive hours every 14 days according to Rosato et al (7). After 4 weeks of treatment she showed healing of digital ulcers and an improvement of RP (reduction of number and severity of attacks).

Two months later, while she still presented RP, she also had asthenia, malar rash, arthralgia, photosensitive rash and oral ulceration. She was normotensive with a normal cardiovascular and respiratory examination. dsDNA antibodies (DNA ELISA 90, NR 0-50 IU/mL), CRP (6 mg/L) and ESR (32 mm/h) were elevated, while complement C3 (0.36, NR 0.9-1.80 g/L) and C4 (0.05, NR 0.10-0.40 g/L) were reduced. ANA were positive at a high titre $(1: 640)$ with a homogeneous pattern and the anti-Sm antibodies were detectable. Other anti-ENA (Scl70, RNP, SSA, SSB, Jo-1) were undetectable. Lupus anticoagulant (LAC), anticardiolipin $\beta 2$ glycoprotein I antibodies and anti-cardiolipin IgG/M antibodies were negative. Chest X-ray was normal, while the rest TTE showed was stable and only a mildly elevated systolic pulmonary arterial pressure (PAPs: $32 \mathrm{mmHg}$ ) without pericardial effusion. She also had normochromic, normocytic anaemia (Hb $10.6 \mathrm{~g} / \mathrm{L})$, leukopenia, normal platelet count and APTT, persistent $24 \mathrm{~h}$ proteinuria (1 g per day), presence of red cells, hemoglobin and granular cylinders on urinalysis. Plasma levels of blood urea nitrogen, creatinine were all normal. The renal biopsy showed glomerulonepritis class II according to WHO LN classification.

She was diagnosed with SLE and was treated with oral methylprednisolone (1 mg/kg daily) and monthly i.v. cyclophosphamide $(1,000 \mathrm{mg})$ for 6 months. The clinical course ameliorated over the next two months with regression of asthenia, malar rash, arthralgia, and oral ulceration. During 6 months of therapy progressively dsDNA antibodies, CRP, ESR, complement C3 and C4, serum hemoglobin, returned to normal values.

After six months of interruption of therapy, RP, photosensitive rash, ANA (1 : 640 with immunofluorescence pattern homogeneous), Sm autoantibosies were present, but renal function was normal.

\section{Discussion}

Digital ulcers and gangrene are common skin manifestations of connective tissue diseases, especially systemic sclerosis, although they are relatively rare in SLE. In SLE, there is a predilection for clinical involvement of the joints, skin, kidney, brain, eye, serosa, lung, heart and gastrointestinal tract (8). Although the skin (malar rash, discoid rash, photosensitivity) is a major organ target of SLE, DUs and RP are not included in the diagnostic criteria of SLE. In the literature atypical presentations of SLE with RP have been reported, but to date there is no description of DUs as the initial manifestation of SLE.

Common cutaneous diseases associated with, but not spe- 
cific for, SLE includes vasculitis, livedo reticularis, alopecia, digital manifestations such as periungual telangiectasia, RP, photosensitivity, and bullous lesions. Cutaneous vasculitis (punctuate lesions, palpable purpura, urticaria, ulcers, papules, erythematous plaques and macules) is frequent in SLE patients.

In a literature search, DUs in SLE have not been described. Nevertheless livedo reticularis and skin ulcers, which are often seen on the pre-tibial and ankles, are the most common skin manifestation of primary and secondary antiphospholipid syndrome (APS). Gangrene and necrosis of skin was found in $19 \%$ and $3 \%$ of APS, respectively. Also atherosclerotic changes in the arteries represent another contributing factor to digital gangrene in SLE (3). DUs are an unusual manifestation of SLE and they are typically associated with long disease duration, antiphospholipid antibody presence, gender (male), atherosclerosis, overlap syndrome and vasculitis (9).

Nonetheless, patients with SLE have $25 \%$ to $50 \%$ of developing RP (3). In SLE patients, RP is frequently present and it is associated with pulmonary hypertension (10). Long disease duration, RP and elevated serum CRP were independent predictive factors for SLE to develop digital gangrene (11). In addition lupus nephritis and RP are significant risk factors for vascular thrombosis in SLE patients with positive antiphospholipid antibodies (12).

Capillaroscopy plays a key role in order to differentiate PRP from secondary RP. Actually NVC represents the best method to analyze microvascular damage in autoimmune diseases. The characteristic SLE pattern includes morphological alterations of capillary loops, venular visibility and sludging of blood with variability of capillary loop length. In patients with SLE, major capillary abnormalities were observed, but no specific pattern was noted. Conversely, major capillaroscopic abnormalities (megacapillaries, hemorrhages and avascular areas) are the hallmarks of the SSc capillary pattern. According to major capillaroscopic abnormalities SSc patients were distributed into the appropriate NVC pattern of microangiopathy (early, active, and late).

In SLE patients the association of RP and "SSc like" findings on nailfold capillaroscopy (SSc pattern) may suggest a new SLE subset with subclinical features of SSc. In addition an important relationship between aCL and nailfold capillary changes in patients with SLE is present, suggesting a direct damage of the vascular endothelium by aCL (13). The SSc pattern is rarely present in SLE patients and the recognition of the SSc capillaroscopic patterns might be particularly prognostic for the early detection of the risk of visceral RP (i.e. coronary heart disease).

In this case report digital ulcers appeared as the initial manifestation of SLE in a young woman with elevated PASP and capillaroscopic scleroderma pattern without others contributing factor to DUs (e.g. anti-phospholipid antibody syndrome, atherosclerosis, long disease duration). Using American Rheumatism Association (ARA) criteria for the diagnosis of SLE (presence of 7 of 11 criteria) we can conclude that the present patient is affected by SLE (14). We can exclude both an undifferentiated connective tissue disease and an overlap syndrome.

In conclusion digital ulcers and gangrene are relatively rare in SLE. Atypical presentations of SLE with RP were reported, but to date there has been no description of digital ulcers as the initial manifestation of SLE.

\section{The authors state that they have no Conflict of Interest (COI).}

\section{References}

1. Wigley FM. Clinical practice. Raynaud's Phenomenon. N Engl J Med 347: 1001-1008, 2002.

2. Steen V, Denton CP, Pope JE, Matucci-Cerinic M. Digital ulcers: overt vascular disease in systemic sclerosis. Rheumatology $\mathbf{4 8}$ (Suppl 3): 19-24, 2009.

3. Bhatt SP, Handa R, Gulati GS, et al. Peripheral vascular disease in systemic lupus erythematosus. Lupus 16: 720-723, 2007.

4. Nagai Y, Shimizu A, Suto M, et al. Digital gangrene in systemic lupus erythematosus. Acta Derm Venereol 89: 398-401, 2009.

5. Nagai Y, Shimizu A, Ishikawa O. Successful treatment with bosen$\tan$ for refractory digital ulcers in a patient with systemic lupus erythematosus. J Dermatol 35: 447-451, 2008.

6. Cutolo M, Sulli A, Pizzorni C, Accardo S. Nailfold videocapillaroscopy assessment of microvascular damage in systemic sclerosis. J Rheumatol 27: 155-160, 2000.

7. Rosato E, Borghese F, Pisarri S, Salsano F. The treatment with Nacetylcysteine of Raynaud's phenomenon and ischemic ulcers therapy in sclerodermic patients: a prospective observational study of 50 patients. Clin Rheumatol 28: 1379-1384, 2009.

8. Mills JA. Medical progress: systemic lupus erythematosus. N Engl J Med 330: 1871-1879, 1994.

9. Drenkard C, Villa AR, Reyes E, Abello M, Alarcón-Segovia D. Vasculitis in systemic lupus erythematosus. Lupus 6: 235-242, 1997.

10. Kasparian A, Floros A, Gialafos E, et al. Raynaud's phenomenon is correlated with elevated systolic pulmonary arterial pressure in patients with systemic lupus erythematosus. Lupus 16: 505-508, 2007.

11. Liu A, Zhang W, Tian X, Zhang X, Zhang F, Zeng X. Prevalence, risk factors and outcome of digital gangrene in 2684 lupus patients. Lupus 18: 1112-1118, 2009.

12. Choojitarom $K$, Verasertniyom $O$, Totemchokchyakarn $K$, Nantiruj K, Sumethkul V, Janwityanujit S. Lupus nephritis and Raynaud's phenomenon are significant risk factors for vascular thrombosis in SLE patients with positive antiphospholipid antibodies. Clin Rheumatol 27: 345-351, 2008.

13. Cutolo M, Sulli A, Secchi ME, Paolino S, Pizzorni C. Nailfold capillaroscopy is useful for the diagnosis and follow-up of autoimmune rheumatic diseases. A future tool for the analysis of microvascular heart involvement? Rheumatology 45: 43-46, 2006.

14. Tan EM, Cohen AS, Fries JF, et al. The 1982 revised criteria for the classification of systemic lupus erythematosus. Arthritis Rheum 25: 1271-1277, 1982.

(C) 2011 The Japanese Society of Internal Medicine http://www.naika.or.jp/imindex.html 